\title{
Flexible PVDF Ferroelectric Capacitive Temperature Sensor
}

\author{
Naveed Khan, Hesham Omran, Yingbang Yao, Khaled N Salama \\ Electrical Engineering Program \\ King Abdullah University of Science and Technology (KAUST) \\ Thuwal 23955, Saudi Arabia \\ Email: khaled.salama@kaust.edu.sa
}

\begin{abstract}
In this paper, a capacitive temperature sensor based on polyvinylidene fluoride (PVDF) capacitor is explored. The PVDF capacitor is characterized below its Curie temperature. The capacitance of the PVDF capacitor changes vs temperature with a sensitivity of $16 \mathrm{pF} /{ }^{\circ} \mathrm{C}$. The linearity measurement of the capacitance-temperature relation shows less than $0.7^{\circ} \mathrm{C}$ error from a best fit straight line. An LC oscillator based temperature sensor is demonstrated based on this capacitor.
\end{abstract}

\section{INTRODUCTION}

Ferroelectric materials are widely used in various applications, such as non-volatile memories, sensors and actuators [1], [2]. Among them, bearing its flexibility nature, polyvinylidene fluoride (PVDF) is recently emerging as a promising material for flexible electronics [3]. Many researchers have tried to integrate PVDF into functional electronic devices, such as sensors and memories [4]. In most cases, the pyroelectric or piezoelectric properties are used in these applications and the material needs to be poled and operated well below its Curie temperature, where the polarization starts to vanish.

On the other hand, temperature sensors are widely used in many applications, such as ambient temperature measurement for environmental monitoring, office and home temperature control, thermal and power management in electronic devices, RFID food monitoring, and temperature compensation of sensory systems [5]-[13]. Historically, CMOS temperature sensors have used substrate PNP BJT transistors to generate a proportional-to-absolute-temperature (PTAT) voltage from the difference in their base-emitter voltages. This approach of temperature measurement needs elaborate calibration and curvature compensation to reduce the non-linearity errors due to the PNP transistors in addition to the need of precision analog interface circuitry which translates to large power consumption [6], [7]. In a standard CMOS process, the native elements such as resistors and MOS transistors have been explored as potential elements to sense the temperature [12], but they suffer from large process variations. A low-power architecture that uses a temperature-to-pulse generator and a time-to-digital converter was proposed in [6] but it has relatively higher inaccuracy compared to BJT-based sensors.

Few reports are found in literature on the evolution of capacitance with temperature as the mechanism for a tem- perature sensor. Capacitive sensors have the advantage of low-power consumption because they do not consume DC current. In addition, the sensor capacitance can be directly digitized to digital output using energy-efficient capacitanceto-digital converter circuitry, and thus avoiding the power consumption, area, and non-idealities of voltage buffers and signal conditioning circuitry [14]-[17].

The dielectric constant of a ferroelectric material changes significantly below its Curie temperature. Such behavior is highly repeatable and moreover, requires no poling for the material itself. In this paper, we use a capacitor made of PVDF, a flexible ferroelectric material as a temperature sensor. The first objective of this paper is to measure the capacitance vs. temperature (CT) behavior below the Curie point (i.e., ferroelectric phase). The other aim of this paper is to explore the feasibility of using the temperature sensitivity of the dielectric constant of PVDF as a means to sense the ambient temperature. The Curie temperature of PVDF is about $180^{\circ} \mathrm{C}$, thus the operating temperature ranges for common electronic devices and biological systems can be adequately covered. Our results show that PVDF is a promising choice for an integrated temperature sensor.

The remainder of the paper is organized as follows. In Section II experimental measurement of CT behavior of PVDF capacitor is demonstrated. In Section III the PVDF capacitor is used to build an LC oscillator circuit where the oscillator output frequency varies with temperature. Experimental measurements of the prototype LC oscillator temperature sensor circuit is presented in Section IV. Finally, conclusion is presented in Section V.

\section{EXPERIMENTAL CT CHARACTERIZATION}

It is well-known that the dielectric constant of a polar material is related to the longitudinal as well as transversal optical phonons of its lattice vibrations, or, the so-called Lydanne-Sachs-Teller (LST) relation [18]. The increase of the dielectric constant with temperature (below Tc) should be related with the relative changes of the longitudinal and transversal optical phonons with temperature. We have used an empirical method to formulate the temperature dependence of the capacitance, by curve-fitting the measured experimental 


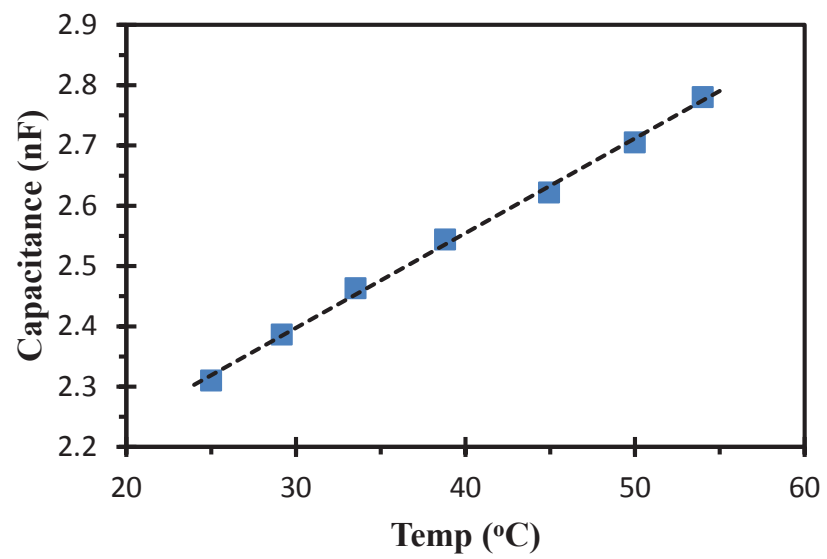

Figure 1. Experimental CT measurements of PVDF capacitor. Measurements were done at $100 \mathrm{KHz}$.

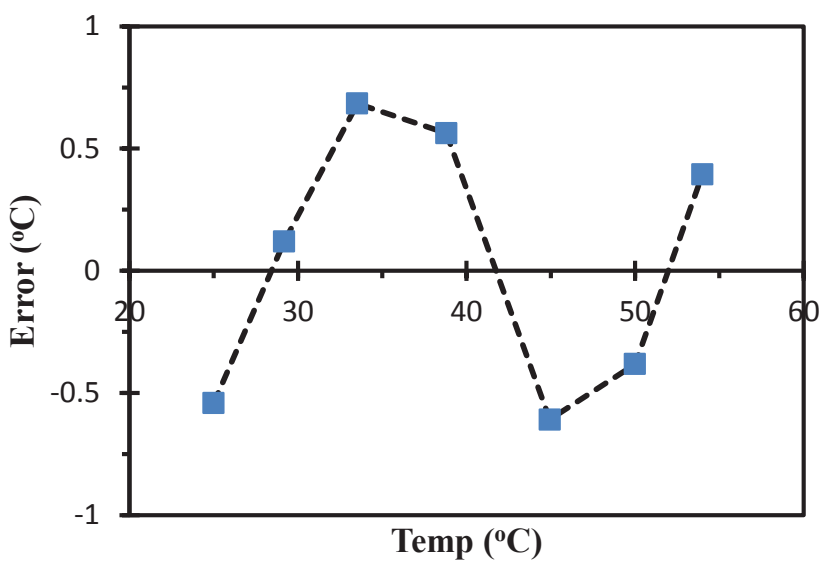

Figure 2. PVDF capacitor linearity error vs temperature.

data. The PVDF capacitor used for this study was characterized Stanford Research Systems (SRS) SR720 LCR. The frequency of the measurement was $100 \mathrm{kHz}$. The temperature of the capacitor was controlled by a Peltier element.

Fig. 1 shows the experimentally measured CT curve of the PVDF capacitor. The capacitor was measured multiple times showing good repeatability. The temperature sensitivity is $16 \mathrm{pF} /{ }^{\circ} \mathrm{C}$. The linearity of the PVDF capacitance was measured by calculating deviation of the CT curve from a straight line; the worst-case deviation from an ideal straight line corresponds to a temperature error of less than $0.7^{\circ} \mathrm{C}$ as shown in Fig. 2 . The change in capacitance due to change in temperature is a linear function, and can be modeled as

$$
C(T)=C_{o}+\alpha \times\left(T-T_{o}\right)
$$

where $C(T)$ is the capacitance at temperature $T$, and $C_{o}$ is the capacitance at a reference temperature $T_{o}$, and $\alpha$ is the sensitivity.

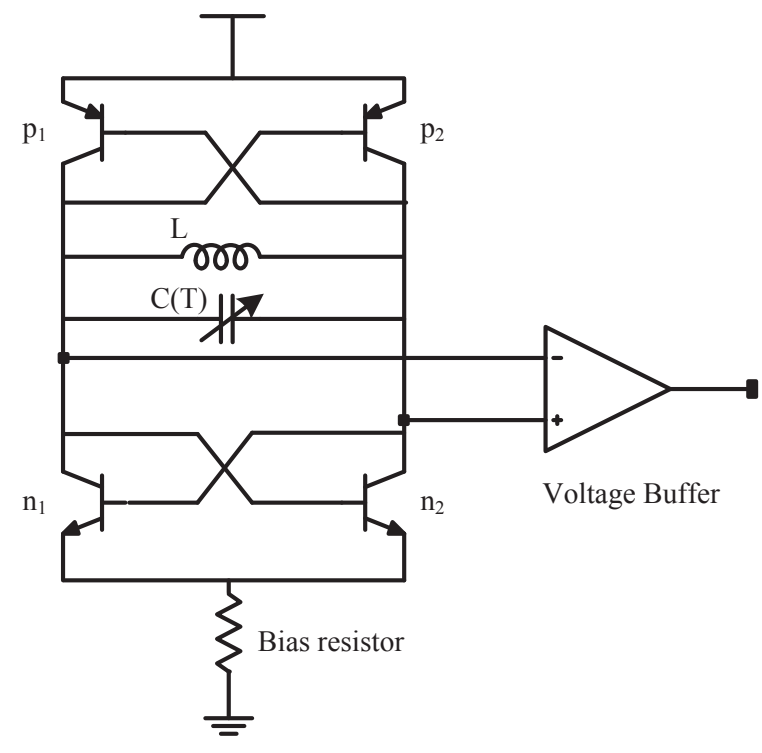

Figure 3. LC oscillator used as a temperature sensor. Components used in experiments: PNP BJTs ( $\mathrm{p} 1$ and $\mathrm{p} 2$ ) are model 9015, NPN BJTs ( $\mathrm{n} 1$ and n2) are model $9013, \mathrm{~L}$ is $0.2 \mu \mathrm{H}, \mathrm{C}(\mathrm{T})$ is the PVDF capacitor used as sensor element where it has a capacitance of $2.3 \mathrm{nF}$ (at $25^{\circ} \mathrm{C}$ ), and the bias resistor is $500 \Omega$.

\section{TEMPERATURE SENSOR CIRCUIT}

To demonstrate the use of PVDF capacitor as a temperature sensor, an LC oscillator circuit shown in Fig. 3 was built. The PVDF capacitor, indicated by $C(T)$ in Fig. 3, is used as the frequency tuning element in the LC oscillator. The LC oscillator is composed of a tuned LC tank, and a negative$g_{m}$, provided by a pair of cross-coupled NPN and PNP BJT transistors. The cross-coupled transistors provide negative- $g_{m}$ to cancel the positive resistance in the LC tank. A voltage buffer was used for isolation to avoid loading effects from measurement instruments.

The complementary cross-coupled oscillator shows a better phase noise performance when compared to the NPN- or PNPonly cross-coupled oscillators for the same supply voltage and bias current (under current limited regime) because of higher amplitude of the oscillation voltage. The oscillation frequency of the LC oscillator at a temperature $T$ is given by

$$
\begin{aligned}
\omega(T) & =\frac{1}{\sqrt{L \times\left[C_{o}+\alpha \times\left(T-T_{o}\right)\right]}} \\
& =\frac{1}{\sqrt{L \times C_{o}}} \times \frac{1}{\sqrt{1+\frac{\alpha \times\left(T-T_{o}\right)}{C_{o}}}}
\end{aligned}
$$

where $\mathrm{L}$ is the inductance; $C_{o}$ is the capacitance of the tank at temperature $T_{o}$, which includes the capacitance of PVDF capacitor, BJTs, and the parasitics from the metal wiring. The capacitance from the BJTs and the parasitic capacitances are ignored in the following analysis. The frequency sensitivity to 
temperature change, is given by

$$
\begin{aligned}
\frac{\delta \omega}{\delta T} & =-\frac{\alpha \times L}{2 \times\left\{L \times\left[C_{o}+\alpha \times\left(T-T_{o}\right)\right]\right\}^{\frac{3}{2}}} \\
& =-\frac{\alpha / C_{o}}{2 \sqrt{L \times C_{o}}} \times \frac{1}{\left\{1+\frac{\alpha \times\left(T-T_{o}\right)}{C_{o}}\right\}^{\frac{3}{2}}}
\end{aligned}
$$

For a temperature change from $T_{o}$ to $T$, the relative change in frequency is given by

$$
\frac{\omega(T)-\omega_{o}}{\omega_{o}}=\sqrt{\frac{C_{o}}{C_{o}+\alpha \times\left(T-T_{o}\right)}}-1
$$

where $\omega_{o}$ is the frequency of oscillation at a reference temperature $T_{o}$.

The PVDF capacitor used as sensor element has a capacitance $C_{o}$ of $2.3 \mathrm{nF}$ at $T_{o}=25^{\circ} \mathrm{C}$. The value of the inductor (L) in Fig. 3 was chosen so that the resonant frequency of the oscillator is about $3.4 \mathrm{MHz}$ at room temperature. NPN 9013 and PNP 9015 discrete BJTs were used to implement the circuit. These BJTs have current gain bandwidth product $\left(f_{T}\right)$ above $100 \mathrm{MHz}$, thus variation of the transistor parameters with temperature will have negligible effect on the oscillation frequency. As can be calculated from (6), for a temperature change from $25^{\circ} \mathrm{C}$ to $70^{\circ} \mathrm{C}$, the change in frequency is expected to be $12.5 \%$

\section{EXPERIMENTAL MEASUREMENTS}

The oscillator circuit was implemented using off-the-shelf components on a printed circuit board. Keithley Nanovoltmeter 2182A and an Agilent 53132A Frequency counter were used for temperature and frequency measurements respectively. The output frequency of the oscillator over $25^{\circ} \mathrm{C}$ to $70^{\circ} \mathrm{C}$ is shown in Fig. 4. With a change of temperature from $25^{\circ} \mathrm{C}$ to $70^{\circ} \mathrm{C}$, the oscillation frequency changes from $3.44 \mathrm{MHz}$ $\left(F_{\max }\right)$ to $3.1 \mathrm{MHz}\left(F_{\min }\right)$. The percent change of frequency, measured as $\frac{F_{\max }-F_{\min }}{F_{\min }}$, is $10.8 \%$, which is slightly lower than the calculated value of $12.5 \%$; the lower percent change in frequency observed in experiments could be attributed to the extra capacitances from the board wiring.

The oscillator spectrum and phase noise measurements were done using Agilent E4448A Spectrum Analyzer. The frequency spectrum of the oscillator over $25^{\circ} \mathrm{C}$ to $70^{\circ} \mathrm{C}$ are shown in Fig. 5. Phase noise measurement of the LC oscillator at $25^{\circ} \mathrm{C}$ shows excellent performance of $-73 \mathrm{dBc} / \mathrm{Hz},-92 \mathrm{dBc} / \mathrm{Hz}$ and $-11 \mathrm{dBc} / \mathrm{Hz}$ at frequency offsets of $1 \mathrm{KHz}, 10 \mathrm{KHz}$ and 100 $\mathrm{KHz}$ respectively; there was negligible phase noise change at $70^{\circ} \mathrm{C}$, which implies the $\mathrm{Q}$ of the PVDF capacitor remained constant over the temperature range.

\section{CONClusion}

The temperature sensitive dielectric-constant of flexible ferroelectric material, PVDF, has been used as a temperature sensor. Temperature sensors built using PVDF capacitors and operated below Curie temperature can provide high measurement

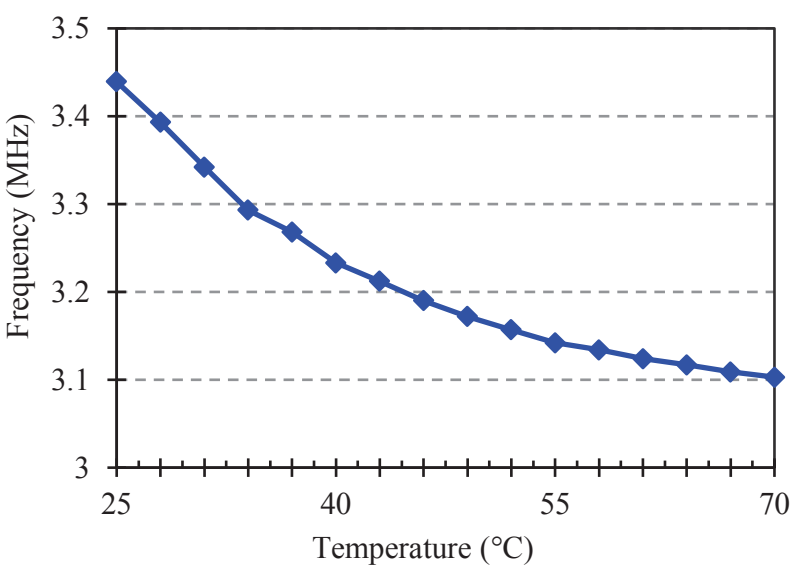

Figure 4. The output frequency of the oscillator shown in Fig. 3 as a function of temperature.

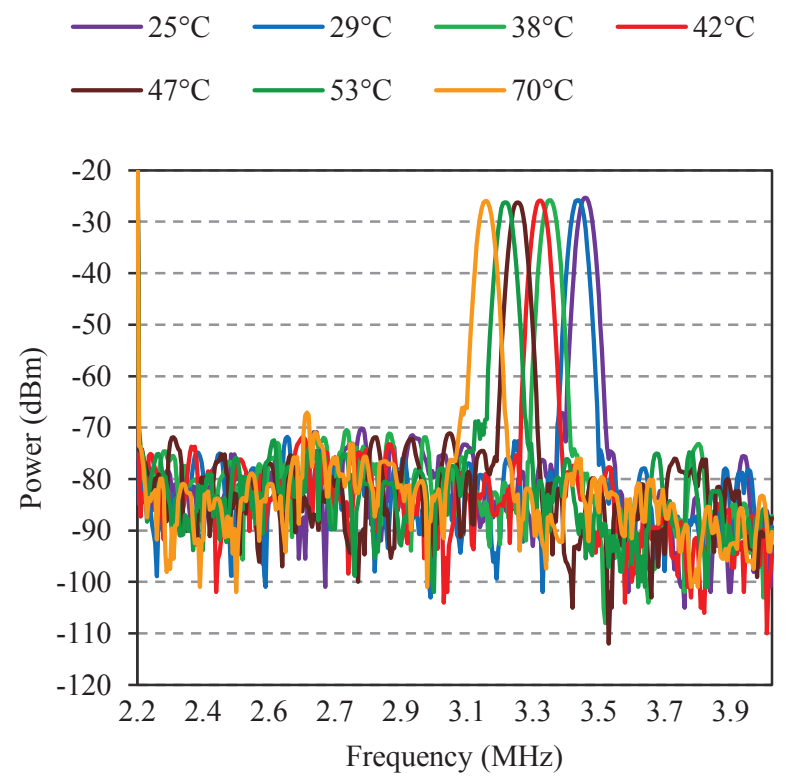

Figure 5. Experimentally measured spectrum of the oscillator over a temperature range $25^{\circ} \mathrm{C}$ to $70^{\circ} \mathrm{C}$.

accuracy because of their linear Capacitance-Temperature relationship. Experimental measurements of PVDF capacitor shows $16 \mathrm{pF} /{ }^{\circ} \mathrm{C}$ sensitivity and less than $0.7^{\circ} \mathrm{C}$ linearity error. Due to their linearity, these sensors can provide high accuracy while minimizing the number of calibrations that are necessary for other types of sensors.

\section{REFERENCES}

[1] J. T. Evans and R. Womack, "An experimental 512-bit nonvolatile memory with ferroelectric storage cell," Solid-State Circuits, IEEE Journal of, vol. 23, no. 5, pp. 1171-1175, 1988.

[2] P. Muralt, "Ferroelectric thin films for micro-sensors and actuators: a review," Journal of Micromechanics and Microengineering, vol. 10, no. 2, p. 136, 2000. 
[3] S. J. Kang, Y. J. Park, I. Bae, K. J. Kim, H.-C. Kim, S. Bauer, E. L. Thomas, and C. Park, "Printable Ferroelectric PVDF/PMMA Blend Films with Ultralow Roughness for Low Voltage Non-Volatile Polymer Memory," Advanced Functional Materials, vol. 19, no. 17, pp. 2812 2818, 2009.

[4] I. Graz, M. Krause, S. Bauer-Gogonea, S. Bauer, S. P. Lacour, B. Ploss, M. Zirkl, B. Stadlober, and S. Wagner, "Flexible active-matrix cells with selectively poled bifunctional polymer-ceramic nanocomposite for pressure and temperature sensing skin," Journal of Applied Physics, vol. 106, no. 3, p. 034503, 2009.

[5] A. Bakker and J. H. Huijsing, High-accuracy CMOS smart temperature sensors. Springer Science \& Business Media, 2000, vol. 595.

[6] P. Chen, C.-C. Chen, C.-C. Tsai, and W.-F. Lu, Solid-State Circuits, IEEE Journal of, vol. 40, no. 8, pp. 1642-1648, 2005.

[7] M. Pertijs, A. Niederkorn, X. Ma, B. McKillop, A. Bakker, and J. Huijsing, "A CMOS smart temperature sensor with a 3 sigma inaccuracy of +-0.5 deg C from -50 deg C to 120 deg C," Solid-State Circuits, IEEE Journal of, vol. 40, no. 2, pp. 454-461, Feb 2005.

[8] P. Chen, C.-C. Chen, Y.-H. Peng, K.-M. Wang, and Y.-S. Wang, "A Time-Domain SAR Smart Temperature Sensor With Curvature Compensation and a 3-sigma Inaccuracy of $-0.4 \operatorname{degC}$ to $+0.6 \mathrm{degC}$ Over a $0 \operatorname{deg} C$ to $90 \operatorname{deg} C$ Range," Solid-State Circuits, IEEE Journal of, vol. 45, no. 3, pp. 600-609, March 2010.

[9] M. Law, A. Bermak, and H. Luong, "A Sub-uW Embedded CMOS Temperature Sensor for RFID Food Monitoring Application," Solid-State Circuits, IEEE Journal of, vol. 45, no. 6, pp. 1246-1255, June 2010.

[10] K. Souri and K. Makinwa, "A $0.12 \mathrm{~mm} 27.4 \mathrm{uW}$ Micropower Temperature Sensor With an Inaccuracy of +/-0.2 degC 3-sigma From - $30 \mathrm{degC}$ to 125 degC," Solid-State Circuits, IEEE Journal of, vol. 46, no. 7, pp. 1693-1700, July 2011.

[11] K. Souri, Y. Chae, and K. Makinwa, "A CMOS Temperature Sensor With a Voltage-Calibrated Inaccuracy of +/-0.15 degC 3-sigma From $55 \operatorname{deg}$ C to 125 degC," Solid-State Circuits, IEEE Journal of, vol. 48 no. 1, pp. 292-301, Jan 2013.

[12] S. A. Jawed, W. A. Qureshi, A. Shafique, J. A. Qureshi, A. Hameed and M. Ahmed, "Low-power area-efficient wide-range robust CMOS temperature sensors," Microelectronics Journal, vol. 44, no. 2, pp. 119 $127,2013$.

[13] S. Jeong, Z. Foo, Y. Lee, J.-Y. Sim, D. Blaauw, and D. Sylvester, "A Fully-Integrated $71 \mathrm{nW}$ CMOS Temperature Sensor for Low Power Wireless Sensor Nodes," Solid-State Circuits, IEEE Journal of, vol. 49, no. 8, pp. 1682-1693, Aug 2014.

[14] Z. Tan, S. Shalmany, G. C. M. Meijer, and M. A. P. Pertijs, "An Energy-Efficient 15-Bit Capacitive-Sensor Interface Based on Period Modulation," Solid-State Circuits, IEEE Journal of, vol. 47, no. 7, pp. 1703-1711, 2012.

[15] H. Omran, M. Arsalan, and K. N. Salama, "7.9 pJ/Step EnergyEfficient Multi-Slope 13-bit Capacitance-to-Digital Converter," Circuits and Systems II: Express Briefs, IEEE Transactions on, vol. 61, no. 8, pp. 589-593, Aug 2014.

[16] —, "A robust parasitic-insensitive successive approximation capacitance-to-digital converter," in Custom Integrated Circuits Conference (CICC), 2014 IEEE Proceedings of the, Sept 2014.

[17] _ " "An integrated energy-efficient capacitive sensor digital interface circuit," Sensors and Actuators A: Physical, vol. 216, pp. 43 - 51, 2014.

[18] W. Cochran, "Crystal stability and the theory of ferroelectricity," Physical Review Letters, vol. 3, no. 9, p. 412, 1959. 